\section{AVANÇOS E DESAFIOS DO ALEITAMENTO MATERNO NO BRASIL: UMA REVISÃO INTEGRATIVA}

\author{
Advances and challenges of breastfeeding in Brazil: an \\ integrative review
}

\author{
Avances y desafios de la lactancia materna en Brasil: una \\ revisión integrativa
}

\section{RESUMO}

Objetivo: Analisar o panorama do aleitamento materno no Brasil, por meio de revisão integrativa da literatura, destacando os seus avanços e desafios. Métodos: Realizou-se revisão da literatura de forma integrativa, nas bases de dados SciELO e PubMed, além de cartilhas publicadas nos sites do Ministério da Saúde e da Rede Internacional em Defesa do Direito de Amamentar (IBFAN), utilizando-se os descritores em português e inglês: aleitamento materno (breastfeeding), autoeficácia (self-efficacy), promoção da saúde (health promotion) e desmame (weaning), no período de 2002 a 2015. Resultados: Identificaram-se inicialmente 43 artigos, 33 cartilhas, 1 dissertação e 3 teses, sendo incluídos no estudo 17 artigos, 3 teses e 19 cartilhas, devido à saturação das informações. Percebeu-se que os índices de aleitamento materno reduziram significativamente ao longo do tempo, com implicações diretas nas taxas de mortalidade infantil, estando associadas ao desmame precoce e à ausência da promoção da autoeficácia materna no pré-natal e no puerpério. Para reverter esse quadro, o Brasil instituiu políticas públicas voltadas para o sucesso da amamentação, que preconizam elevar os indicadores. Conclusão: Apesar dos avanços, o panorama brasileiro relativo à amamentação demonstra que o país permanece aquém das recomendações dos organismos internacionais, constituindo-se num dos grandes desafios da saúde pública brasileira a superação dos obstáculos que impedem o sucesso do aleitamento materno.

Descritores: Aleitamento materno; Autoeficácia; Promoção da Saúde; Desmame.

\section{ABSTRACT}

Objective: To analyze the panorama of breastfeeding in Brazil through an integrative literature review highlighting its advances and challenges. Methods: We carried out an integrative literature review in the SciELO and PubMed databases and in booklets published on the websites of the Ministry of Health and the International Baby Food Action Network (IFBAN) using the following Portuguese and English descriptors: aleitamento materno (breastfeeding), autoeficácia (self-efficacy), promoção da saúde (health promotion) and desmame (weaning) in the period from 2002 to 2015. Results: We identified at first 43 articles, 33 booklets, 1 thesis and 3 dissertations, including in the study 17 articles, 3 dissertations and 19 booklets due to information saturation. It was verified that breastfeeding rates have reduced significantly over time with direct implications in infant mortality rates, being associated with early weaning and the lack of promotion of maternal self-efficacy in the prenatal and postpartum. To change this situation, Brazil imposed a number of public policies aimed at breastfeeding success, which advocated to raise rates. Conclusion: Despite the advances, the Brazilian panorama of breastfeeding shows that the country remains below the recommendations of international organizations, and overcoming the obstacles to successful breastfeeding constitutes a major challenge for the Brazilian public health.

Descriptors: Breastfeeding; Self-efficacy; Health Promotion; Weaning.
Artigo de Revisão

Francisca de Sousa ${ }^{(1)}$

Maísa de Lima Claro ${ }^{(1)}$

Andréia Lima de Sousa ${ }^{(1)}$

Luisa Helena de Oliveira Lima ${ }^{(1)}$

Andrea Gomes da Silva

Santana $^{(1)}$

1) Universidade Federal do Piauí - UFPI Picos (PI) - Brasil

Recebido em: 09/09/2015

Revisado em: 16/09/2015

Aceito em: 29/09/2015 


\section{RESUMEN}

Objetivo: Analizar el panorama de la lactancia materna en Brasil a través de una revisión integrativa de la literatura destacando sus avances y desafios. Métodos: Se realizó una revisión de la literatura de manera integrativa en las bases de datos SciELO y PubMed, además de cartillas publicadas en las páginas web del Ministerio de la Salud y de la Red Internacional en Defensa del Derecho de Amamantar (IBFAN), utilizándose los descriptores en portugués e inglés: lactancia materna (breastfeeding), autoeficacia (self-efficacy), promoción de la salud (health promotion) y destete (weaning) en el periodo entre 2002 y 2015. Resultados: A principio se identificaron 43 artículos, 33 cartillas, 1 disertación y 3 tesis, siendo inclusos en el estudio 17 artículos, 3 tesis y 19 cartillas debido la saturación de las informaciones. Se percibió que los índices de lactancia materna se redujeron significativamente a lo largo del tiempo con implicaciones directas en las tasas de mortalidad infantil, asociándose con el destete precoz y la ausencia de la promoción de la autoeficacia materna en el prenatal y el puerperio. Para revertir ese cuadro, Brasil ha instituido politicas públicas dirigidas al éxito de la lactancia materna que preconizan la subida de los indicadores. Conclusión: A pesar de los avances, el panorama brasileño de lactancia materna muestra que el país sigue abajo de las recomendaciones de los organismos internacionales, constituyéndose en uno de los grandes desafios de la salud pública brasileña la superación de los obstáculos que impiden el éxito de la lactancia materna.

Descriptores: Lactancia Materna; Autoeficacia; Promoción de la Salud; Destete.

\section{INTRODUÇÃO}

A presença de indicadores insatisfatórios do aleitamento materno tem acarretado grandes problemas à saúde pública, em especial no que diz respeito aos índices de morbimortalidade infantil, sendo consenso na literatura que a amamentação previne, de forma isolada, inúmeras mortes infantis. Das 10 milhões de mortes de crianças que são registradas anualmente no mundo, um milhão e meio delas seriam evitadas mediante o aumento da cobertura para $90 \%$ de aleitamento materno exclusivo nos seis primeiros meses de vida nos países em desenvolvimento ${ }^{(1)}$, mas os dados divulgados pela UNICEF no ano de 2013 apontam que somente $39 \%$ das crianças menores de seis meses recebem aleitamento materno exclusivo ${ }^{(2)}$.

Entre os aspectos relacionados à redução nas taxas do aleitamento materno (AM) no Brasil, destaca-se o papel do marketing instituído pelas empresas de fórmulas infantis, que, entre outras práticas, realizavam a distribuição gratuita de seus produtos para as mães dos recém-nascidos, disseminando a cultura de que a alimentação artificial era melhor que o AM. Outros fatores apontados na literatura para a queda nesses índices foram os processos de industrialização e urbanização, a inserção da mulher no mercado de trabalho e a cultura do uso da mamadeira, que apresentava um atributo simbólico de modernidade ${ }^{(3)}$.

Diante da constante queda nos índices de aleitamento materno, o Ministério da Saúde no Brasil traçou linhas de ação, como a Área Técnica de Saúde da Criança e Aleitamento Materno / Departamento de Ações Programáticas Estratégicas (DAPES) / Secretaria de Atenção à Saúde (SAS), voltada para a promoção, proteção e apoio ao aleitamento materno, uma vez que essa tríade contribui para reduzir a mortalidade infantil, além de possibilitar ao Brasil cumprir as metas firmadas com os organismos internacionais ${ }^{(4)}$.

Essas metas foram acordadas na Declaração do Milênio, no ano de 2000, como meio de modificar o cenário mundial em relação à pobreza alarmante, em que uma a cada três pessoas sobreviviam com menos de um dólar por dia. Dentre as metas, referem-se à saúde materno-infantil: a redução da mortalidade na infância e melhoria da saúde materna, com prazo de cumprimento estabelecido para 2015. Segundo essas metas, deveria ocorrer a redução da mortalidade infantil para 17,9 óbitos a cada mil nascidos vivos e melhorar os indicadores da saúde materna, atingindo a razão de mortalidade igual ou menor que 35 óbitos para 100 mil nascidos vivos $^{(5)}$.

Nesse aspecto, já no ano de 2011 o Brasil atingiu um dos Objetivos do Milênio, ao reduzir 2/3 da mortalidade infantil entre os anos de 1990 e 2015, obtendo taxa de mortalidade infantil de 15,7 óbitos. Essa redução foi proporcional em todas as regiões brasileiras, porém, a região Nordeste foi a que apresentou um maior percentual de decréscimo nessas taxas, em torno de $6,6 \%$ ao ano ${ }^{(6)}$.

Diante desse cenário, fica nítida a relevância da promoção do aleitamento materno, que proporciona, entre seus inúmeros benefícios, a redução na incidência das doenças infecciosas e alergias, levando a uma diminuição da morbimortalidade infantil, ao garantir um aporte nutricional adequado para a criança. Além disso, reduz os riscos de a mãe vir a desenvolver alguns tipos de câncer e anemia, faz com que o intervalo entre futuras gestações sejam prolongados e promove satisfação maior em relação às mulheres que não amamentam ${ }^{(7)}$.

Além do papel do aleitamento materno na redução da mortalidade infantil, ele tem sido apontado como fator importante de proteção à saúde do indivíduo em longo prazo. As pesquisas têm comprovado que previne o sobrepeso e a obesidade durante toda a infância e na vida adulta, estando implicado no processo do imprinting metabólico, que se refere à experiência nutricional precoce do indivíduo em períodos críticos e pontuais, levando a um efeito prolongado 
e constante ao longo de toda sua existência, além de tornálo suscetível a algumas doenças ${ }^{(8,9)}$.

Estudo recente ainda aponta que a prática da amamentação exerce efeitos cognitivos. Crianças que são amamentadas por um período maior de tempo refletiriam níveis de inteligência e renda média mais elevadas na fase $\operatorname{adulta}^{(10)}$.

Diante da importância do aleitamento materno, tem sido frequente o estudo dos fatores envolvidos na sua efetivação. De maneira mais recente, tem ganhado destaque a autoeficácia, que diz respeito à confiança materna em amamentar, o que influencia diretamente na prática e duração do aleitamento materno ${ }^{(11)}$.

Nesse sentido, a confiança materna em praticar a amamentação é construída sobre quatro tipos de informações, entre elas: a experiência pessoal bem sucedida no período de aleitamento anterior, através de momentos vivenciados que estimulem a amamentação; o fato de receber orientações e apoio de pessoas próximas, que incentivem a prática do aleitamento; e apresentar bom estado emocional e fisiológico que contribuam para o sucesso do ato de amamentar ${ }^{(12)}$.

Diante da relevância do aleitamento materno na efetivação da saúde materno-infantil, bem como na prevenção de doenças crônicas não transmissíveis, o objetivo do presente estudo foi analisar o panorama do aleitamento materno no Brasil, por meio de revisão integrativa da literatura, destacando os seus avanços e desafios.

\section{MÉTODOS}

Para a elaboração do presente artigo, realizou-se revisão bibliográfica de forma integrativa por meio de busca eletrônica nas bases de dados do Scientific Electronic Library Online (SciELO) e U.S. National Library of Medicine and the National Institutes Health (PubMed), além de consulta às cartilhas e outras publicações de relevância no portal do Ministério da Saúde, no Instituto Econômico de Pesquisa Aplicada e no site da Rede Internacional em Defesa do Direito de Amamentar (IBFAN), sendo a busca realizada entre setembro de 2014 e abril de 2015.
$\mathrm{Na}$ pesquisa dos artigos, utilizaram-se os seguintes descritores, obtidos junto aos Descritores em Ciências da Saúde (DeCS: http://decs.bvs.br): aleitamento materno (breast feeding), autoeficácia (self-efficacy), promoção da saúde (health promotion) e desmame (weaning). Dependendo da base de dados utilizada, os termos foram utilizados em português e/ou inglês.

A pesquisa ocorreu entre setembro de 2014 e abril de 2015, e incluiu artigos e cartilhas que abordavam a temática do aleitamento materno no Brasil, tendo sido selecionados aqueles que enfatizavam os seguintes aspectos: indicadores do aleitamento materno, autoeficácia, causas do desmame precoce e políticas públicas instituídas no território nacional em prol da promoção, proteção e apoio à amamentação.

Constaram ainda como critérios de inclusão: publicações datadas entre os anos de 2002 e 2015, sendo incluídas duas referências de 1975 e 1977, por tratarem de conteúdos relevantes para a compreensão do trabalho; terem sido realizadas somente em humanos e publicadas nos idioma inglês e português. Excluíram-se as publicações que apresentavam inconsistência com a temática do estudo, informações duplicadas e cujo texto não estivesse disponível na íntegra.

\section{RESULTADOS}

Na primeira busca, selecionaram-se 80 trabalhos, dos quais são 43 artigos, 33 cartilhas disponíveis nos sites do Ministério da Saúde e da Rede Internacional em Defesa do Direito de Amamentar e do Instituto Econômico de Pesquisa Aplicada, além de 1 dissertação e 3 teses.

Do total de 80 trabalhos analisados, percebeu-se que menos da metade, $48,75 \%(n=39)$, estava em conformidade com a temática que seria investigada. Após a leitura e avaliação do conteúdo, foram incluídos na atual revisão 17 artigos, devido à saturação das informações, além de serem adicionadas 3 teses e 19 cartilhas, pois tinham uma matriz teórica fundamentada nos objetivos do presente estudo.

Em súmula, os estudos selecionados, no tocante aos indicadores do aleitamento no Brasil e ao papel da autoeficácia materna para sua promoção, não apresentam conformidades em relação às metodologias, uma vez que

Tabela I - Percentual dos indicadores de aleitamento materno no Brasil segundo as regiões, de acordo com a II Pesquisa de Prevalência sobre o Aleitamento Materno, realizada no ano de $2008^{(15)}$.

\begin{tabular}{cccccccccc}
\hline \multicolumn{4}{c}{ Percentual de AME por regiões } & \multicolumn{5}{c}{ Percentual de AM por regiões } \\
\hline NO & CO & S & SE & NE & NO & CO & S & SE & NE \\
$45,9 \%$ & $45 \%$ & $43,9 \%$ & $39,4 \%$ & $37 \%$ & $76,9 \%$ & $64,1 \%$ & $59,1 \%$ & $51,4 \%$ & $49,5 \%$ \\
\hline
\end{tabular}

Fonte: Venancio; Escuder; Saldiva; Giugliani, 2010. 
a temática é ampla e cada autor enfatiza um determinado tópico, correlacionando com as variáveis de interesse, sendo isso uma das dificuldades em comparar os estudos e ter de fato a real dimensão desses indicadores.

Além do mais, a autoeficácia é um tema recente, que vem sendo investigado aos poucos no Brasil. Esse fato é comprovado quando se analisa o período em que foram publicados os estudos: o ano de 2002 foi tido como ponto de partida, mas somente a partir do ano de 2010 é que houve maior número de publicações, cerca de 56,41\% ( $\mathrm{n}=22)$.

Porém, notou-se que, no Brasil, só haviam sido executados trabalhos utilizando a escala da autoeficácia em três cidades brasileiras: Fortaleza, Caxias do Sul e São Paulo. A primeira, localizada na região Nordeste; a segunda, na região Sul; e a terceira, na região Sudeste do país, o que não permite traçar um perfil fidedigno, uma vez que são cidades com características sociodemográficas muito diferenciadas.

\section{DISCUSSÃO}

\section{Indicadores do aleitamento materno no Brasil}

Até a década de 1980, havia uma escassez muito grande de registros referentes à prevalência do aleitamento materno no Brasil, e foi somente a partir de então que as pesquisas passaram a ser realizadas, começando a evidenciar a sua relevância e verificando a necessidade de uma padronização no momento de coletar os dados, para proporcionar uma maior confiabilidade dos resultados ${ }^{(13)}$.

No entanto, uma pesquisa que demonstrasse de fato a realidade dos indicadores de aleitamento materno (AM) no Brasil só foi realizada em 1999 pelo Ministério da Saúde, sendo os dados coletados em todas as capitais brasileiras, exceto no Rio de Janeiro. Após esse período, o Brasil só veio a realizar estudo de âmbito nacional no ano de 2008 , com a II Pesquisa de Prevalência do Aleitamento Materno, dessa vez realizada em todas as capitais brasileiras e no Distrito Federal, sendo assim possível traçar um paralelo dos indicadores avaliados ao longo de quase dez anos, identificando os índices positivos e negativos, e possibilitando delinear novas metas ${ }^{(4)}$.

Cabe salientar que os dados de 2008 são os mais recentes e, em 2014, foi realizado um estudo piloto a fim de validar uma nova metodologia que possa mensurar os índices do AM, sendo o estudo realizado em três capitais (Rio de Janeiro, Recife e Brasília). Talvez, em 2015, possa ser ampliado para todas as capitais do Brasil, com a meta de alcançar a faixa etária de crianças menores de dois anos e a inclusão da alimentação complementar saudável. Após essa etapa, espera-se que, em 2016, consiga-se aplicar a pesquisa de Demografia e Saúde (DHS), após anos da última coleta ${ }^{(14)}$.
A partir dos resultados obtidos na pesquisa de 2008, constatou-se que o aleitamento materno exclusivo (AME) em crianças menores de seis meses ficou em torno de $41 \%$ na soma das capitais e DF. Já quando essa prevalência foi analisada por capital, verificou-se que a região Norte possuía a maior taxa de AME; e o Nordeste, a menor taxa, conforme Tabela I ${ }^{(15)}$.

Ao comparar a prevalência do AME, no período de 1999 a 2008, na população brasileira, notou-se um aumento na sua duração média, com elevação de um mês, passando de 23,4 para 54,1dias, demonstrando um avanço positivo na incidência dessa prática ${ }^{(15)}$.

Com relação à análise do $\mathrm{AM}$ nas crianças de 9 a 12 meses, foi encontrada uma prevalência de $58,7 \%$, com a região Norte continuando a apresentar os melhores indicadores, conforme Tabela I. Fazendo um paralelo entre os anos de 1999 e 2008, notou-se que a prevalência do AM passou de 295,9 para 341,6 dias, havendo um aumento de aproximadamente $45 \operatorname{dias}^{(15)}$.

Ficou constatado, nessas duas pesquisas, que a região Norte apresentou os melhores índices e que o Brasil melhorou muito suas taxas de amamentação ao longo desses quase dez anos. A melhoria nos indicadores esteve associada à elevação da escolaridade materna, da idade das mulheres quando decidem engravidar e do tempo de licença maternidade, que em alguns casos chega aos seis meses. Mesmo com os avanços registrados, o país ainda encontrase longe do cumprimento das metas estabelecidas pela OMS e MS para a prática do $\mathrm{AME}^{(4)}$.

A discrepância nos valores de prevalência, tanto do AME quanto do AM, nas diferentes regiões deve-se ao fato de o Brasil ser um país com um território muito extenso e rico em diversidades ${ }^{(16)}$.

\section{Causas do desmame precoce}

O desmame consiste na oferta de outros alimentos que não o leite materno para a criança, indo desde a introdução de chás e água, culminando com a retirada total da amamentação ${ }^{(17)}$. Entre os fatores que contribuem para a ocorrência desse episódio, há aqueles que vêm sendo estudados ao longo dos tempos, os quais se denominam clássicos, tais como: a mãe possuir trabalho informal ou estar desempregada, o grau de escolaridade, a idade materna, a alegação de pouco leite, o grau de aceitação e planejamento da gravidez, o vínculo afetivo com o parceiro e a influência da mãe nessa escolha ${ }^{(18)}$.

Em um estudo realizado em municípios da região norte do estado de São Paulo, observou-se que o fato de a mãe possuir trabalho informal ou estar desempregada esteve entre os determinantes do desmame precoce, mas que o grau de escolaridade e a idade materna não contribuíram de 
forma significativa para a efetivação dessa prática, além de não se verificar influência das condições socioeconômicas maternas na determinação do desmame precoce ${ }^{(19)}$. No entanto, duas outras pesquisas ${ }^{(20,21)}$ observaram correlação com o tempo de estudo materno, verificando-se que as nutrizes que possuíam menos de oito anos de escolaridade ou o primeiro e segundo grau incompletos apresentaram uma maior incidência de desmame quando comparadas às mães que estudaram por mais tempo. No entanto, só foi demonstrada associação com a idade materna em um estudo(21), no qual as mães com menos de 20 anos apresentaram prevalência maior do desmame.

Apesar de os estudos destacarem diversos fatores influenciando o desmame precoce, ainda prevalece a alegação de pouco leite, que em sua maioria está relacionada a causas não confiáveis. Por isso, é de extrema importância analisar a presença dos reais indicativos da baixa produção láctea, que são a redução da diurese e do número de evacuações (com alterações de suas características), e o ganho de peso insatisfatório para idade ${ }^{(22)}$.

Embora estejam bem estabelecidos os fatores que atuam classicamente na interrupção do aleitamento materno, as políticas públicas não têm sido efetivas no seu controle, o que pode ser constatado a partir dos indicadores nacionais da amamentação, os quais permanecem inferiores às recomendações. Dessa forma, tem-se buscado a identificação de outros determinantes do desmame precoce, que, apesar de presentes, apresentam difícil reconhecimento, sobretudo por seu caráter subjetivo, tendo sido frequente o esforço em criar estratégias para quantificá-los. Nesse aspecto, destacase o nível de confiança materna em sua capacidade para amamentar ${ }^{(23)}$.

Para mensuração desse parâmetro, foi desenvolvida uma escala cujo somatório das respostas resulta em número variável de escores. A interpretação desse instrumento demonstra que um escore elevado é um indicativo de que o período de aleitamento materno exclusivo tende a ser maior em relação a valores menores ${ }^{(24)}$.

Diante das evidências apresentadas, pode-se afirmar a relevância da identificação dos fatores determinantes do desmame precoce, da forma como interagem entre si, bem como de estratégias de enfrentamento deles, uma vez que podem atuar para a melhoria do estado nutricional das crianças e pelos impactos que apresentam na saúde infantil ${ }^{(20)}$

\section{Políticas públicas de promoção, proteção e apoio ao aleitamento materno}

A partir da constatação dos prejuízos advindos do desmame precoce, a Organização Mundial da Saúde e o Fundo das Nações Unidas firmaram parcerias entre os diversos países visando à redução nas taxas de mortalidade infantil. No Brasil, a primeira iniciativa aconteceu na década de 1980, com a implantação do Programa de Incentivo ao Aleitamento Materno ${ }^{(4)}$.

Depois dele, surgiram várias outras políticas públicas de promoção, proteção e apoio ao AM; dentre elas, destacam-se a Iniciativa Hospital Amigo da Criança ${ }^{(25)}$; a Norma de Comercialização de Alimentos para Lactentes de Primeira Infância, Bicos, Chupetas e Mamadeiras ${ }^{(1)}$ e a Lei $11.265 / 2006^{(1)}$; o Método Mãe Canguru ${ }^{(26)}$; a Rede Cegonha $^{(27)}$, que contempla a Estratégia Amamenta e Alimenta Brasil (28); além das Semanas de Aleitamento Materno $^{(29)}$ e das Salas de Apoio à Amamentação ${ }^{(30)}$.

A Iniciativa Hospital Amigo da Criança (IHAC) foi lançada no ano de 1991, tendo como objetivo oferecer para as mães e seus filhos recém-nascidos um momento agradável para a amamentação, de maneira a possibilitar o aumento das chances do AME durante os seis primeiros meses de vida e a continuidade de uma alimentação para a criança, com a oferta de alimentos complementares saudáveis, incentivando a conciliação com a amamentação até os dois anos ou mais. Por isso, para o alcance desses objetivos, implantaram-se como condicionalidade os Dez Passos para o Sucesso do Aleitamento Materno. São recomendações que devem ser seguidas por toda unidade que preste serviço de maternidade e cuidado neonatal ${ }^{(25)}$.

Para amparar legalmente e complementar os Dez Passos para o Sucesso do Aleitamento Materno, foi criada a NBCAL, Norma Brasileira de Comercialização de Alimentos para Lactentes e Crianças de Primeira Infância, Bicos, Chupetas e Mamadeiras, e a Lei 11.265/2006, que regulariza a comercialização e o marketing realizados pelas indústrias de alimentos, mamadeiras e bicos. A regulamentação da divulgação desses produtos contribui para a proteção da amamentação ${ }^{(1)}$.

Nessa perspectiva, o Ministério da Saúde lançou, ainda, o programa Método Mãe Canguru, o qual consiste na assistência neonatal especializada, que estimula o contato precoce pele a pele entre a mãe e o bebê recém-nascido de baixo peso, contribuindo para aumentar o vínculo afetivo e, consequentemente, estimular o aleitamento materno. Isso colabora para a elevação das taxas de frequência, duração e precocidade do AM, além de reduzir a ocorrência de infecção hospitalar ${ }^{(26)}$.

Já a Rede Cegonha foi criada para desempenhar ações voltadas para as mulheres que visam garantir um atendimento gratuito de qualidade, seguro e humanizado, através de assistência que atinge o planejamento familiar, o pré-natal, o parto e o puerpério, indo até os dois anos de vida da criança. Esse acompanhamento tem por finalidade reduzir os índices de mortalidade materna e infantil, além 
de garantir um nascimento seguro e um crescimento e desenvolvimento de forma saudável ${ }^{(27)}$.

Uma das principais estratégias da Rede Cegonha é a Rede Amamenta e Alimenta Brasil, política lançada em 2012 através da integração da Rede Amamenta Brasil e da Estratégia Nacional para a Alimentação Complementar Saudável (ENPACS), com o compromisso de formar recursos humanos na atenção básica, visando à qualificação desses profissionais, para que reforcem e incentivem a promoção do AM e a alimentação saudável para crianças menores de dois anos no âmbito do Sistema Único de Saúde $(\mathrm{SUS})^{(28)}$.

Outra iniciativa de destaque é a Semana Mundial de Aleitamento Materno, que consiste em campanha cuja temática possui abrangência internacional, mas cada país adota uma metodologia específica de desenvolvimento das estratégias, de acordo com sua realidade ${ }^{(29)}$.

As mulheres ainda podem contar com as Salas de Apoio à Amamentação, regulamentadas pela nota técnica conjunta número 01 de 2010, cuja elaboração é datada de março de 2009. Por meio dessa iniciativa, as empresas disponibilizam um espaço para que as nutrizes possam realizar a retirada do leite materno de maneira confortável, com privacidade e segurança durante o expediente, além de possuir um local refrigerado específico para guardar os recipientes e, ao final do dia, levá-los para casa e oferecêlos aos seus filhos ou, se preferirem, doar para um banco de leite humano. Além disso, toda empresa que possuir no seu quadro de funcionários mais de 30 mulheres acima dos 16 anos deverá ter uma creche apropriada para que as crianças fiquem alocadas durante todo o período de amamentação ${ }^{(30)}$.

Além das políticas e iniciativas mencionadas, a legislação brasileira disponibiliza como aparato legal de proteção ao aleitamento materno a licença maternidade, que em seu texto original previa 120 dias de afastamento remunerado para as mães trabalhadoras e o direito às grávidas de não serem demitidas sem justa causa, a partir do momento da confirmação da gestação até cinco meses após o parto, além de poderem contar com duas pausas diárias de trinta minutos destinados para amamentarem seus filhos até que estes completem seis meses de idade. A partir de 2010 o Congresso Nacional prorrogou a licença maternidade para 180 dias, por meio do Programa Empresa Cidadã, mediante a concessão de incentivos fiscais ${ }^{(31)}$.

No caso de mães estudantes, a legislação ainda é incipiente, pois a lei que a regulamenta, datada da década de 1970, prevê apenas 90 dias de afastamento ${ }^{(32)}$. Para os pais, a Constituição ainda garante a licença paternidade de cinco dias, a contar do dia do nascimento do filho, com o objetivo de fazer o registro civil do recém-nascido e organizar a chegada do bebê em casa ${ }^{(30)}$.
Apesar de as políticas em prol da amamentação terem progredido, não se pode afirmar, mediante dados estatísticos, se estão sendo eficazes na elevação dos índices de AM em todo o território brasileiro, pois os indicadores disponíveis não acompanharam a evolução delas, sendo necessário aguardar novos estudos para constatar se a tendência de elevação desses índices ocorreu de fato ${ }^{(14)}$.

\section{Autoeficácia na amamentação}

A autoeficácia na amamentação diz respeito ao nível de confiança que a mãe possui na sua capacidade de amamentar. Nesse aspecto, convém destacar que se encontra bem estabelecida na literatura a teoria da autoeficácia de Bandura $^{(33)}$, segundo a qual qualquer ação que o ser humano venha a desempenhar sempre passa pela autoeficácia, pois o indivíduo tem a necessidade de sentir-se encorajado a encarar os diferentes obstáculos que irão surgir todos os dias na sua vida.

Com base nessa teoria, e diante da necessidade de mensuração de fatores passíveis de intervenção que possam explicar a interrupção precoce do aleitamento materno, um estudo desenvolveu e validou, em 1999, no Canadá, a escala Breastfeeding Self-Efficacy Scale ${ }^{(34)}$. A relevância da aplicação desse método para averiguação da confiança materna em praticar a amamentação permite o delineamento de estratégias de intervenção nos pontos de maior vulnerabilidade de cada nutriz.

Diante da sua importância, traduziu-se, adaptou-se e validou-se a escala ao idioma português ${ }^{(33)}$, sendo conduzido um estudo com gestantes da cidade de Fortaleza, no estado do Ceará ${ }^{(23)}$. O teste final foi aplicado em 117 gestantes e obteve resultados satisfatórios, indicando a eficácia da escala para a identificação de pontos específicos do nível de confiança materna em amamentar, tanto em nível de pesquisa quanto clínico, viabilizando aos profissionais da saúde atuar nos itens em que as gestantes apresentem menor confiança, permitindo-lhes intervir e reduzir os índices de desmame precoce ${ }^{(23,34)}$.

A validação nacional da escala Breastfeeding SelfEfficacy Scale ${ }^{(34)}$ tem permitido o desenvolvimento de importantes trabalhos em todo o país, em diferentes estágios da lactação, a exemplo do realizado em um estudo ${ }^{(35)}$ no sul do Brasil entre nutrizes. Os resultados dessa investigação demonstraram que escores elevados de autoeficácia estiveram relacionados à prática do aleitamento materno exclusivo. Já no estudo realizado com 100 puérperas em um hospital privado da cidade de São Paulo, utilizando-se a escala Breastfeeding Self-Efficacy Scale - Short Form (BSES-SF), os escores para a autoeficácia em praticar o aleitamento materno foram elevados na maioria das mães investigadas $(82,3 \%)$, porém, não foi constatada diferença 
estatisticamente significativa quando comparada a média de tempo de aleitamento exclusivo e não exclusivo com os escores de média e alta eficácia ${ }^{(36)}$.

Outro desdobramento da adaptação desse instrumento de pesquisa ao contexto brasileiro consistiu no desenvolvimento do álbum seriado "Eu posso amamentar o meu filho", elaborado e validado ${ }^{(37)}$, cujo embasamento teórico encontra-se nas questões abordadas na escala BSESSF. Nesse estudo com 201 puérperas em uma maternidade pública no município de Fortaleza, Ceará, elas foram subdividas em dois grupos: controle e intervenção. Este último recebeu orientações sobre o AM através do álbum seriado e obteve uma elevada autoeficácia materna em relação à amamentação, o que acarretou um maior tempo no AM.

Dessa forma, passa-se a dispor de dois importantes referenciais na busca de melhoria dos indicadores de aleitamento materno: a escala de autoeficácia, que permite a identificação dos pontos de vulnerabilidade materna em sua habilidade de amamentar, e o álbum seriado "Eu posso amamentar o meu filho", a ser utilizado para elevar os escores de autoeficácia da amamentação, que se traduz em aumento da confiança da mãe na sua capacidade de iniciar e manter o aleitamento materno. Resultado de estudo recente ${ }^{(11)}$ reafirma a importância desses instrumentos, verificandose que a utilização do referido álbum seriado teve grande impacto sobre as nutrizes, pois, após a intervenção, estas demonstraram mais confiança e determinação em praticar a amamentação.

Nota-se, a partir das investigações, que a utilização de metodologias que partem do princípio da teoria da autoeficácia tem grandes impactos sobre a duração do $\mathrm{AM}$, pois permite identificar os pontos mais fragilizados de gestantes e nutrizes, devendo-se reforçar a confiança materna, de modo a evitar o desmame precoce $^{(38)}$.

\section{CONCLUSÃO}

Conclui-se que o panorama brasileiro em relação à amamentação apresenta-se deficitário, apesar dos esforços empreendidos em prol da sua valorização.

No tocante aos indicadores do aleitamento materno, estes ainda se encontram aquém do recomendado pelos organismos internacionais. Quanto às políticas públicas instituídas, estas partem de princípios bem fundamentados, porém, precisam ser mais efetivas na consolidação de uma rede de apoio à amamentação, o que incide na qualificação dos profissionais de saúde para incentivarem a prática do aleitamento materno e estimularem a autoeficácia da mulher em praticar a amamentação, uma vez que está comprovado que esse fator é decisivo, porque desencoraja o desmame precoce.
Com isso, percebe-se a necessidade da realização constante de estudos que visem analisar periodicamente esse panorama, de modo a direcionar as ações dos órgãos e profissionais de saúde, para que tenham maior efetividade e, consequentemente, mais indivíduos possam usufruir dos benefícios do aleitamento materno.

\section{REFERÊNCIAS}

1. Rede Internacional em Defesa do Direito de Amamentar, Ministério da Justiça. Secretaria de Direito Econômico, Conselho Federal Gestor do Fundo de Defesa dos Direitos Humanos. Alimentos para crianças de até 3 anos, bicos, chupetas e mamadeiras. São Paulo: Ministério da Justiça; 2007.

2. UNICEF. State of the World's Children. Nova York: UNICEF; 2013.

3. Oliveira MIC, Souza IEO, Santos EM, Camacho LAB. Avaliação do apoio recebido para amamentar: significados de mulheres usuárias de unidades básicas de saúde do Estado do Rio de Janeiro. Ciênc Saúde Coletiva. 2010;15(2):599-608.

4. Ministério da Saúde (BR), Secretaria de Atenção à Saúde, Departamento de Ações Programáticas e Estratégicas. Pesquisa de Prevalência de Aleitamento Materno em Municípios Brasileiros: situação do Aleitamento Materno em 227 municípios brasileiros. Brasília: Ministério da Saúde; 2010.

5. Instituto de Pesquisa Econômica Aplicada. Objetivos de Desenvolvimento do Milêno: relatório nacional de acompanhamento. Brasília: Ipea; 2010.

6. Ministério da Saúde (BR), Secretaria de Atenção Básica à Saúde. Indicadores de Desenvolvimento Brasileiro. Brasília: Ministério da Saúde; 2013.

7. Ministério da Saúde (BR), Secretaria de Atenção à Saúde. Departamento de Atenção Básica. Saúde da criança: nutrição infantil: aleitamento materno e alimentação complementar. Brasília: Ministério da Saúde; 2009.

8. Balaban G, Silva GAP, Dias MLCM, Fortaleza GTM, Morotó FMM, et al. O aleitamento materno previne o sobrepeso na infância? Rev Bras Saúde Matern Infant. 2004;4(3):263-8.

9. Simon VGN, Souza JMP, Souza SB. Aleitamento materno, alimentação complementar, sobrepeso e obesidade em pré-escolares. Rev Saúde Pública. 2009;43(1):60-9.

10. Victora CG, Horta BL, Mola CL, Quevedo L, Pinheiro RT, Gigante DP, et al. Association between breastfeeding 
and intelligence, educational attainment, and income at 30 years of age: a prospective birth cohort study from Brazil. Lancet Glob Health. 2015;3(4):199-205.

11. Dodt RCM, Ferreira AMV, Nascimento LA, Macêdo AC, Joventino ES, etal.Influência de estratégia de educação em saúde mediada por álbum seriado sobre a autoeficácia materna para amamentar. Texto \& Contexto Enferm. 2013;22(3):610-8.

12. Edficher M. Fatores associados ao desmame precoce: percepção de autoeficácia no aleitamento materno e depressão pós-natal [tese]. Porto Alegre: Pontíficia Universidade Católica do Rio Grande do Sul; 2013.

13. Ministério da Saúde (BR), Secretaria de Atenção à Saúde, Departamento de Ações Programáticas e Estratégicas. II Pesquisa de Prevalência de Aleitamento Materno nas Capitais Brasileiras e Distrito Federal. Brasília: Ministério da Saúde; 2009.

14. Müller FS, Rea MF, Monteiro NR. Iniciativa mundial sobre tendências do aleitamento materno (Wbti): informe nacional. São Paulo: IBFAN Brasil; 2014.

15. Venancio SI, Escuder MML, Saldiva SRDM, Giugliani ERJ. A prática do aleitamento materno nas capitais brasileiras e Distrito Federal: situação atual e avanços. J Pediatr (Rio J). 2010;86(4):317-24.

16. Sena MCF, Silva EF, Pereira MG. Prevalência do aleitamento materno nas capitais brasileiras. AMB Rev Assoc Med Bras. 2007;53(6):520-24.

17. Ministério da Saúde (BR), Secretaria de Atenção à Saúde, Departamento de Atenção Básica. Política Nacional de Alimentação e Nutrição. Brasília: Ministério da Saúde; 2003.

18. Dodt RCM, Oriá MOB, Pinheiro AKB, Almeida PC, Ximenes LB. Perfil epidemiológico das puérperas assistidas em um alojamento conjunto. Rev Enferm UERJ. 2010;18(3):354-1.

19. Venancio SI, Escuder MML, Kitoko P, Rea MF, Monteiro CA. Frequência e determinantes do aleitamento materno em municípios do Estado de São Paulo. Rev Saúde Pública. 2002;36(3):313-8.

20. Volpini CCA, Moura EC.Determinantes do desmame precoce no distrito noroeste de Campinas. Rev Nutr. 2005;18(3):311-9.

21. França GVA, Brunken SMS, Silva, SM, Escuder MM, VenancioSI.Determinantes da amamentação no primeiro ano de vida em Cuiabá, Mato Grosso. Rev Saúde Pública. 2007;41(5):711-8.
22. Ministério da Saúde (BR), Secretaria de Atenção à Saúde, Departamento de Ações Programáticas e Estratégicas. Atenção à saúde do recém-nascido: guia para os profissionais de saúde. Brasília: Ministério da Saúde; 2011.

23. Oriá MOB, Ximenes LB. Tradução e adaptação cultural da Breastfeeding Self-EfficacyScale para o português. Acta Paul Enferm. 2010;23(2):230-8.

24. Dennis CL. The Breastfeeding Self-Efficacy Scale: psychometric assessment of the short form. J Obstet Gynecol Neonatal Nurs. 2003;32(6):734-44.

25. Ministério da Saúde (BR), Fundo das Nações Unidas Para a Infância. Iniciativa Hospital Amigo da Criança: revista, atualizada e ampliada para o cuidado integrado: módulo 1: histórico e implementação. Brasília: Ministério da Saúde; 2008.

26. Ministério da Saúde (BR), Secretaria de Políticas Públicas da Saúde, Área de Saúde da Criança. Atenção humanizada ao recém-nascido de baixo peso: método mãe canguru: manual do curso. Brasília: Ministério da Saúde; 2002.

27. Ministério da Saúde (BR), Secretaria de Políticas Públicas da Saúde, Área de Saúde da Criança. Rede Cegonha. Brasília: Ministério da Saúde; 2010.

28. Ministério da Saúde (BR), Secretaria de Políticas Públicas da Saúde, Área de Saúde da Criança. Estratégia Amamenta e Alimenta Brasil. Brasília: Ministério da Saúde; 2012.

29. Ministério da Saúde (BR), Secretaria de Políticas Públicas da Saúde, Área de Saúde da Criança. Semanas Mundiais de Aleitamento Materno. Brasília: Ministério da Saúde; 2012.

30. Ministério da Saúde (BR), Agência Nacional de Vigilância Sanitária, Área Técnica de Saúde da Criança e Aleitamento Materno, Departamento de Ações Estratégicas. Nota Técnica Conjunta $n^{\circ} 01 / 2010$ : Salas de Apoio à Amamentação em Empresas. Brasília: Ministério da Saúde; 2010.

31. Ministério da Saúde (BR), Área Técnica de Saúde da Criança e Aleitamento Materno, Departamento de Ações Estratégicas. Licença maternidade. Brasília: Ministério da Saúde; 2010.

32. Brasil. Lei $\mathrm{n}^{\circ}$ 6202, de 17 de abril de 1975. Dispõe sobre a Licença Maternidade para Estudantes. Diário Oficial da União 1975 Abr 17.

33. Bandura A. Self-efficacy: toward a unifying theory of behavioral change. Psychol Rev. 1977;84(2):191-215. 
34. Oriá MOB. Tradução, adaptação e validação da Breastfeeding Self-EfficacyScale: aplicação em gestantes [tese]. Fortaleza: Universidade Federal do Ceará; 2008.

35. Zubaran C, Foresti K. The correlation between breastfeeding self-efficacy and maternal postpartum depression in southern Brazil. Sexual and Reproductive Healthcare. 2013;4(1):9-15.

36. Souza EFC, Fernandes RAQ. Autoeficácia na amamentação: um estudo de coorte. Acta Paul Enferm. 2014;27(5):465-70.

37. Dodt RCM. Elaboração e Validação de tecnologia educativa para autoeficácia da amamentação [tese]. Fortaleza: Universidade Federal do Ceará; 2011.
38. Dodt RCM, Javorski M, Nascimento LA, Ferreira AMV, Tupinambá MC, Ximenes LB. Álbum seriado sobre aleitamento materno: intervenção educativa com nutrizes no pós-parto imediato. Rev Enferm UFPE on line. 2013;7(5):1469-75.

\section{Endereço para correspondência:}

Artemízia Francisca de Sousa

Universidade Federal do Piauí (UFPI)

Campus Senador Helvídio Nunes de Barros

Rua Cícero Eduardo s/n Bairro Junco

CEP: 64.600-000 - Picos -PI - Brasil

E-mail: artesousa@hotmail.com 